\title{
Cutaneous Anthrax surveillance by Person, Place, and Time in Georgia (2008-2013)
}

\author{
Anna Kasradze ${ }^{3}$, Khatuna Zakhashvili ${ }^{3}$, Diana Echeverria ${ }^{1,5}$, Nicholos Heyer ${ }^{1}$, David \\ Garcia $^{\star 1,2}$, Ian Kracilik ${ }^{4}$ and Paata Imnadze ${ }^{3}$
}

${ }^{1}$ Battelle Memorial Institute, Bakersfield, CA, USA; ${ }^{2}$ University of North Carolina, Chapel Hill, NC, USA; ${ }^{3}$ National Center for Disease Control and Public Health, Tbilisi, Georgia; ${ }^{4}$ University of Florida Gainesville, Gainesville, FL, USA; ${ }^{5}$ University of Washington, Seattle, WA, USA

\section{Objective}

To characterize descriptive trends using data collected by the national Electronic Integrated Disease Surveillance System (EIDSS, 2008-2013).

\section{Introduction}

Cutaneous anthrax is endemic in Georgia ${ }^{1}$. The EIDSS program captures notifications from 72 municipal public health centers. It links urgent notification, case investigation data, and laboratory data on an online basis. Eleven virulent and 4 non-virulent strains of $B$. anthracis have been isolated. Genotype $G_{K} 35$ and $G_{K} 44$ are strains found in the Turkish-Southern Caucasian region. It is hypothesized that human rates are caused by increased contact with infected animals. The recent re-introduction of animal vaccination programs in 2013-2014 heightens interest is establishing a defensible 6-year baseline trend in humans.

\section{Methods}

Data from all investigations of suspect, potential, and laboratory confirmed cases of cutaneous Anthrax were obtained from EIDSS from $01 / 01 / 2008$ to $12 / 31 / 2013$. The NCDCPH laboratory network confirmed diagnoses. Samples were plated on PLET and SDA media and final confirmation was performed using real-time PCR (Roche Light Cycler 2.0 using B. anthracis Target 2 and B. anthracis Target 3 Idaho Technology kits). DNA isolation was completed using Qiagen DNeasy Blood \& Tissue Mini Kits. A descriptive data analysis was performed by person, place, and time. Census data was provided by the National Statistics Office of Georgia.

\section{Results}

Among 634 notifications, 343 were confirmed cases (54\%), 136 were probable cases $(21 \%)$, and 9 were suspect cases $(1 \%)$. Laboratory testing eliminated $22.0 \%$ and $86 \%$ were confirmed by bacteriology and PCR, 3.4\% by PCR and $10.6 \%$ by bacteriology.

Number and Incidence Rates (IR) per 100,000. The 500 cases were examined annually where $82 \%$ were male between 20 to 60 years of age suggesting infection maybe occupationally related. Also $38 \%$ of cases reported contact with animal products, $29 \%$ cleaned farms, and $12 \%$ had contact with soil. The 6-year mean IR among Georgian nationals $(\mathrm{n}=290)$ was 1.32 and annual rates were 1.45 , $0.79,0.57,1.56,1.69$, and 1.86. The rate for Azerbaijani nationals were 9.31 which increased from $2.11,1.76$, to $3.16,7.73,23.88$, and 17.21. The contrast was pronounced in Kvemo Kartli (Gargabani $\mathrm{IR}=45.49$ vs 14.21; Marneuli/Tsalka IR=20.10 vs 7.61), in Kakheti (Sagarejo IR=23.28 vs 4.06), and in Samtskhe-Javakheti (Bojormi and Bakuriani $I R=3.17$ vs 0.51 ) where a large proportion of Azerbaijani pastoralists lives and migrates along sheep and cattle corridors.

Variation by Year and Week in the Year. Cases appeared to increase from 2010 to 2013, but only cases for the Azerbaijani minority group met statistical significance. Overall statistical differences were also observed for the mean number of cases in 2008-2010 from that in 2011-2013. The 3-year means in Weeks 21-29 and in Weeks 39-44 increased respectively from 0.79 to 4.39 in spring and from 0.92 to 3.22 in the fall.

\section{Conclusions}

The evidence is consistent with the conclusion that cutaneous anthrax cases are secondary to epizootic events more likely occurring in populations that work with unvaccinated sheep and cattle. The recent increase in cases is more related to a true increase in disease rather than improved reporting because incidence rates remained steady in Georgian nationals and only increased in Azerbaijani minorities that work and live along animal corridors. Higher occupational risks are also supported by higher rates observed in 20 to 60 year old males as well as independently computed odds ratios of 6.1 (2.1-17.7 95\% CI) for working in animal husbandry ${ }^{2}$. Pending approval, the NCDCPH proposes a national state program designed to conduct active surveillance in pastoralists. The evidence also underscores the importance of other One Heath recommendations to activate anthrax awareness campaigns in Russian and Azerbaijani, supervise the destruction of known carcasses, record GIS sites and disinfect infected soils, and introduce a participatory health education tool on anthrax.

\section{Keywords}

Cutaneous anthrax; Georgia; Azerbaijanis; Pastoralists

\section{Acknowledgments}

We would like to thank Mariam Broladze who served as our disease coordinator.

\section{References}

Kracalik I, Malania L, Tsertsvadze N et al. 2014. PLoS Negl Trop Dis. Jul 2014; 8(7): e2985.

Navdarashvili A. 2013. FELTP EIS AOG Meeting. Atlanta Georgia.

*David Garcia

E-mail: garciadvd77@gmail.com 